\title{
AN UNMANNED AERIAL VEHICLE BASED FOREST PATROL SYSTEM
}

\author{
Songsheng LI \\ Department of Computer Engineering, \\ Guangdong College of Business and Technology, Zhaoqing, China
}

\begin{abstract}
Although technology has advanced at breathless pace, wildfire still bursts out every year all over the world. There are various wildfire monitoring system employed in diferrent countries, most of them are depended on photos or videos to identify features of wildfire, after wildfire happened. Delay of confirmation is diverse based on used techonologies. An autonomous forest patrol system by Unmanned Aerial Vehicle $(U A V)$ is presented in this paper, which try to employ the fashinable UAV to patrol in forest and collect environmental data in order to monitor and predict wildfire before it really erupts. From limited practical data collected, the monitoring data such as temperature and humidity are effective to reflect the real situation, prediction requires more data and time to prove.
\end{abstract}

\section{KEYWORDS}

UAV, Bluetooth, Forest, Wildfire, Patrol

\section{INTRODUCTION}

Autonomous forest patrol is a classical topic as forest occupy almost $31 \%$ surface of the earth in 2012 according to EARTH POLICY INSTITUTE from Rutgers University [1]. In general, forests are not under surveillance, it is too wide for human to cover even parts of it before. Modern invention such as airplane and robot arouse possibility of forest exploration. Satellites [2] are employed to monitor wildfire, but they only help after burning. PETER KOURTZ [3] wrote the paper named "A VISUAL AIRBORNE FOREST FIRE DETECTION PATROL ROUTE PLANNING SYSTEM" when working in Canadian Forest Service in 1973. The scene of the paper is that a pilot flies an airplane in a planned route, observes the forest on the ground for sign of wildfire. Route is significant because of budget restriction and time limitation if wildfire happens; it is planed according to fire occurrence pattern from historical data. As there is no real computer at that time, all the test was done almost by hand. Comparably, UAV of today is tiny, flexible and intelligent, should be used effectively. Luis Merino et al. proposed an Unmanned Aircraft System for wildfire monitoring [4], which employ several aerial vehicles and a central station, vehicles collaborate and automatically obtain wildfire information by means of on-board infrared or visual camera. Every vehicle equips with local perception and software component; they make decision on the real-time progress of the fire front shape, applying techniques of prediction model, fire contours extraction, image vibrations eliminating, feature matching etc. Their experiment showed that fire front shape is adequately extracted from the images, and estimation of the fire front shape was successful, the system is applicable. At least two issues should be stressed, one is scale, their experiment employed 3 vehicles, but the coverage area is limited to visual range of people. As more than one vehicle is used, collaboration is another problem, the system need visual photos from different vehicles, so how to organize their

David C. Wyld et al. (Eds) : CNDC, DPPR, AIAA, WiMNET, ACITY - 2017

pp. 01- 10, 2017. (C) CS \& IT-CSCP 2017

DOI : $10.5121 /$ csit.2017.71601 
synchronization and path to get useful vision is key factor of their success. It will turn out to be very complex if more than one wildfire bursts.

M. Hefeeda and M. Bagheri present the design and evaluation of a wireless sensor network (WSN) for early detection of forest fires [5]. They focus on the Fire Weather Index (FWI) from the Canadian Forest Service, treat the forest fire detection problem as a k-coverage problem in WSN. In addition, they present a simple data aggregation scheme to prolong the network lifetime by only delivering the data of interest to the application. Their simulation way is far from practice, first is deployment, with increase of $\mathrm{k}$, the massive nodes are need based on the algorithm, and nodes die quickly.

Paper [6] describes a hierarchical WSN for early fire detection in risky areas. Advantage it elaborates includes 1) no communication network in advance, 2) time from the GPS module, 3) not just fire early detection, environment monitoring too, 4) no cameras/images, 5) middleware layer to share data. There are some questions left of the paper, first is the time synchronization, which is not described how to synchronize time from CNs to SNs, and relevant energy consumed. Second is battery of SNs, it was said that battery with a capacity of $600 \mathrm{mAh}$ can last at least 2 years without prove.

Habiboglu et al. proposed a real-time video smoke detection system that uses correlation descriptors with an SVM classifier [7]. They use temporally extended correlation matrices to combine color, spatial and temporal information together in the decision process. They proved that the proposed method is computationally efficient. Duration for the algorithm to identify the wildfire is the most crucial index for wildfire detection. The algorithm needs three stages, 1) slow moving object detection in video, 2) smoke-colored region detection, 3) correlation based classification. So time is related to performance of camera and computer.

This paper [8] presents the system architecture, hardware and software framework of WSN based wildfire monitoring system. They claimed main contribution of the paper is the design can meet the goal of reactivity and reliability, robustness and network lifetime. Specifically, reactivity depends on threshold, reliability needs diagnostic phase and link quality based routing protocol, robustness achieves with water-and-fire-proof boxes, network lifetime is extended by a MAC protocol named SMAC and dynamic power management. Left issues are listed as 1) balance of coverage and cost, 2) location information of data is not solved, 3) how will the mobile station work.

Comparison in [9] summarizes four main ways, human based observation, satellite system, optical cameras and WSNs, to forest fire detection in seven aspects including cost, efficiency and practicality, faulty alarms repetition, fire localizing accuracy, detection delay, small fire behavior information, can be used for other purposes. They all have advantage and disvantage, but optical cameras and WSN are relatively preferred.

Titled "Use of Remote Sensing in Wildfire Management" [10], the chapter elaborates wildfire management in a total different way for pre-fire and post-fire conditions monitoring, which is space-borne remotely sensed imagery, consist of optical remote sensing, thermal infrared remote sensing and radar remote sensing. Authors admit that further studies are required to establish models to estimate fuel moisture, and further to assess optical and thermal infrared images, radar images for monitoring pre-fire conditions too. Other required input for monitoring fire danger, like wind parameters, cannot be derived from remote sensing data. And geo-stationary satellites provide a lower revisit time ( 1 to 2 daily passes) so it is not ideal for real-time monitoring of wildfire. 
Lina Tang and Guofan Shao reviewed applications of drone in forestry research and practice [11]. They first distinguished drone remote sensing from crewed aircraft remote sensing and satellite remote sensing then some projects employed drone successfully, such as surveying, mapping, measuring, tracking of forest situation, and supporting intensive forest management etc. They agree that drones are very susceptible to weather and human-related accident, and multidisciplinary collaborations to promote the standardization of drone is iminent.

We present a localization framework of WSN [12] [13] using dynamic path of mobile beacon (DPMB). The imaged scenario of the application is that an unmanned vehicle (UV) such as UAV or a driverless car, moves on the field where massive sensor nodes are deployed, its moving path will be decided dynamically by information from nodes. UV is the master of algorithm behind, it starts with first triple positions to ensure all nodes inside the triangle be converted to reference nodes, then it walks on vertex of equilateral triangle, in every position, "flood 3" employs reference to localize more nodes, then "Try all 2" checks nodes only received 2 messages based on their localized neighbors, and those two steps are repeated until no new nodes are localized, then UV moves to next position. we adapted online Reinforcement Learning (RL) as brain of UV to drive UV on the field to perform localization. According to the principle of exploration or exploitation, UV could regret the first choice and have a second chance to localize more nodes [14]. An alternative metric, direction, specifically, Vector Cosine Similarity (VCS) instead of distance is employed when decision on neighbours [15]. Nodes are grouped and grouped weight is calculated, which UV is driven by. Simulation proves that algorithms are lightweight and effective with help of UAV.

In this paper, the forest patrol system is implemented by UAV, observation points (OP) are distributed in forest, their locations are marked when installation, UAV fly to them according to the locations and receive data by Bluetooth. Before Bluetooth 4.1 [16], a Bluetooth Low Energy (BLE) device is either master or slave, cannot be both. This is how BLE used in the system, a UAV with a BLE data agent (BLEDA) acts as a mobile central, collects data from peripheral, which is BLE data collector (BLEDC) in OPs.

\section{FOREST PATROL SYSTEM}

The proposed forest patrol system is based on the currently popular UAV and combined with BLE to implement automatic forest patrol and predict the possible eruption of wildfire.

\subsection{System Introduction}

The system is displayed in figure 1, there are some OPs are selected in the surveilled forest; UAV stops at station usually and flies along a fixed and scheduled flight route which covers all the OPs and starts from station and ends at station. All the detail about OP, UAV and station are described in the following.

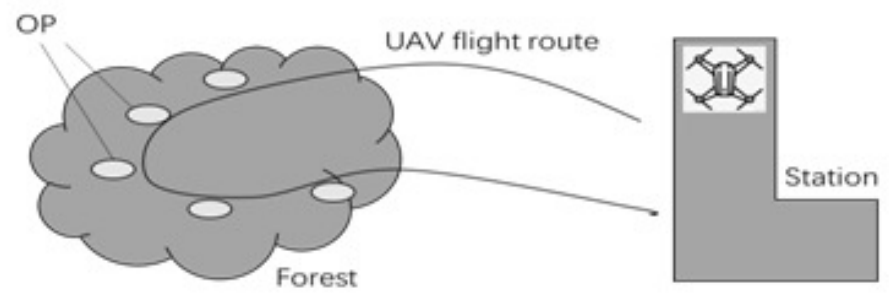

Figure 1. Introduction 


\subsubsection{Selection of $O P$}

OP should be chosen in the middle of the surveilled forest, where that part of forest is important or vulnerable, then a BLE data collector (BLEDC) attached with a solar panel will be installed, which is a box with sensors embedded in surface. It will collect various sensor data continuously and dynamically, and report to UAV regularly. GPS information of the OP will be recorded when installation.

\subsubsection{UAV Collecting Data}

UAV equipped with a BLEDA will fly to OPs according to the GPS information in storage, which are recorded at installation stage. BLEDA will connect to BLEDCs, receive collected sensor data, return to station, and report data to cloud database. This is the routine task of UAV in the system. Two features of Intelligent Flight Modes [17] of UAV are very helpful for our system, one is waypoints, they are multiple GPS points which are set in control part of UAV. If they are set, the UAV will automatically fly to them, for our system, those points are OPs. The other is point of interest (POI), if a point is set in the control of UAV as POI, UAV will continuously circle around it for information.

\subsubsection{Station}

Station is the base for UAV, it provides resources the system requires, which include database server, Wi-Fi router, recharge for UAV and BLEDA, etc. There will be shelter for UAV and staffs.

Two key components in the system are BLEDC and BLEDA, they are explained in the following.

\subsection{BLEDC}

BLEDC is responsible for collecting sensor data and reporting to UAV, whose structure is shown in Figure 2. As it is installed in severe wild environment, its structure is strictly specified.

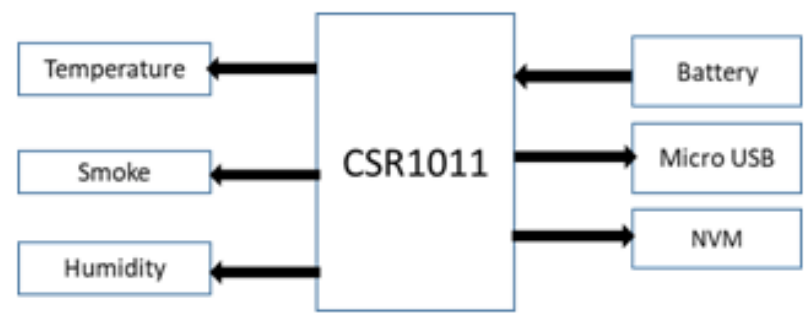

Figure 2. BLEDC

Three main sensors, temperature, humidity and smoke detector are integrated to IO of CSR1011 which is a decent single-mode BLE product from CSR. It collects data from those sensors dynamically, attaches timestamp, saves to non-volatile memory (NVM) and reports to BLEDA when it comes. Some specific details:

BLEDC plays role of GATT server in BLE architecture. According to BLE standard [18], as a GATT server, BLEDC must setup at least one Service with Universally Unique Identifier (UUID) and inside this Service there is at least one Characteristic with UUID too. Different BLE devices can be identified by this specific Service UUID. BLEDA use this UUID to find BLEDC. 
Cyclic storage is designed for the application, for example, 512 bytes are applied from NVM, a head pointer for read and a tail point to write are defined, when head reach tail, no new data is available, when tail reach head, no more space available. This is the flexible and general way in practice.

Dynamical data means recording data base on threshold which is a value between normal and abnormal. Data beyond threshold will be recorded more frequently than normal. In such way significant data are recorded and normal data could be ignored, as a result, saving power and memory of BLEDC.

CSR1011 is designed by consideration of power exhaust, only a small 3v button battery CS2032 can provide energy of months, but for the system solar panel is a better choice if it is feasible, in other words, if the OP can provide enough sunshine for the panel.

In general, BLEDC only executes slow advertising based on schedule, but in special situation such as value from sensor change dramatically, it will keep slow adverting to open possibility for connection from BLEDA in order to report emergence on time.

Taking the wild bad climate and environment into consideration, the whole equipment adopts engineering plastics and sensors are embedded on the surface to promise them expose to the true environment, and all the related materials are chosen strictly to meet the industry requirement. No matter solar panel is used or not, save energy is the first principle as overcast could last long enough to exhaust solar power.

\subsection{BLEDA}

It is carried by UAV to approach OP to receive data from BLEDC. Its structure is shown in Figure 3. The core of BLEDA is ESP32, which is a Wi-Fi and Bluetooth combo chip, all the data reported from BLEDCs will be uploaded to database on Station by Wi-Fi. A GPS chip is integrated to the equipment to provide accurate time and location information. Storage employs the similar circular management way as BLEDC. Power supply in BLEDA will be very flexible as UAV can return to Station to recharge. LED applies different colors and flash to represent various working situation. Long press of button will power on or off the equipment and short press will change mode of BLEDA. There are two working modes designed for BLEDA, one is setup mode, the other is client mode.

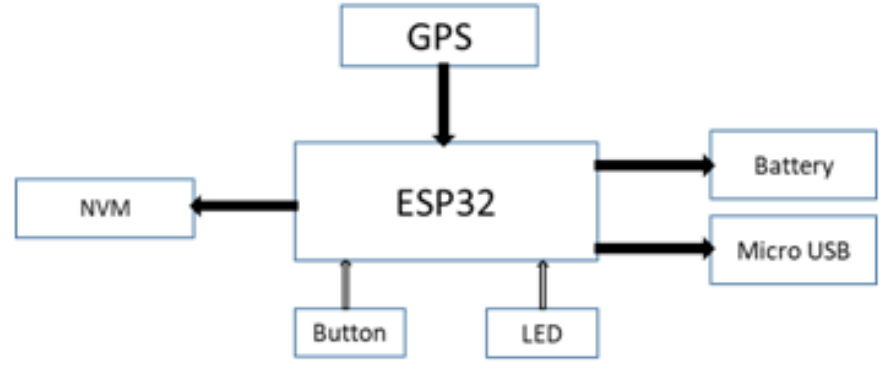

Figure 3. BLEDA

The purpose of setup mode is to check the GPS information of OPs. UAV carries BLEDA and flies on the route of patrol to Ops after all the GPS information of Ops are set to UAV as waypoints. When flying, BLEDA starts BLE and search for BLE device which owns Service with UUID of this system, if BLEDA is connected and reports information, Bluetooth address and GPS information of every BLEDA are saved into NVM. 
The client is the general mode of BLEDA. It is called client because BLEDC is defined as GATT Server in Bluetooth 4 and BLEDA is GATT client. Its main task is receiving data from BLEDC. In flight, BLEDA keeps tracking GPS information, compare it with GPS information of BLEDCs from NVM, if they are similar or close, then open BLE and connect to the BLEDC and receive data and save them to NVM.

\subsection{System Setup Procedure}

The procedure is shown in Figure 4. The flow chart explains itself and the detail of Bluetooth communication is hidden. Slow advertising is a way for BLEDC to notice BLEDA that it is waiting for connection. Compared with its counterpart, fast advertising, it saves energy, but it is a waste of energy if it keeps advertising. This could be a one-time effort if anything goes well. This procedure makes sure that the information of BLEDCs is consistent with the original selection of Ops. Application in Station server will compare the information BLEDA collected with records of Ops, if any difference emerges, all human error and performance of UAV should be ruled out first, then coverage range of BLE should be taken into consideration as it can be affected by environment remarkably.

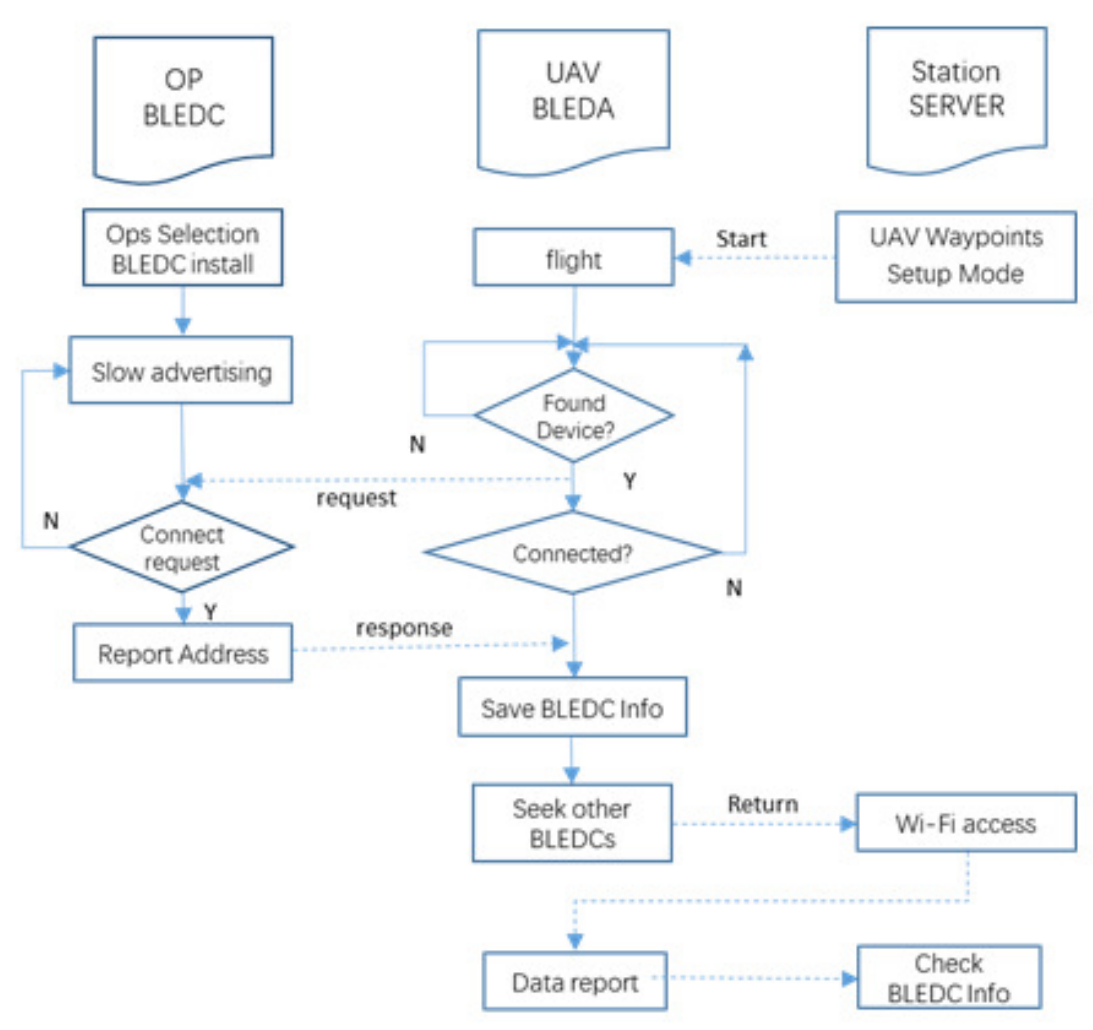

Figure 4. Setup procedure

\subsection{Routine Procedure}

Compared to setup procedure, the routine deserves more elaboration, which is displayed in Figure 5. The significance of time synchronization, scheduled slow advertising and exception handle are explained below. 


\subsubsection{Time Synchronization}

System clock of CSR1011 is based on crystal oscillator which is not absolutely accurate, its error will be larger with time last longer, so it should be corrected with accurate time from GPS. So a significant step of routine procedure is time synchronization, which is happened right after BLEDA connected to BLEDC, BLEDC receives time by the way of concerted protocol, updates its own time which will be used from then on, and uploads sensor data to BLEDA. So the timestamp of data from first routine could be deflected.

\subsubsection{Scheduled Slow Advertising}

In routine procedure, scheduled slow advertising is employed instead of slow adverting which is employed in setup procedure. The former is scheduled, so it only slow advertising in certain time with certain interval, and the latter will always advertise in a slow way. The necessity of the former is power efficiency which is key topic in WSN, it is possible that cloudy and rainy weather could last long then power from solar panel exhausts.

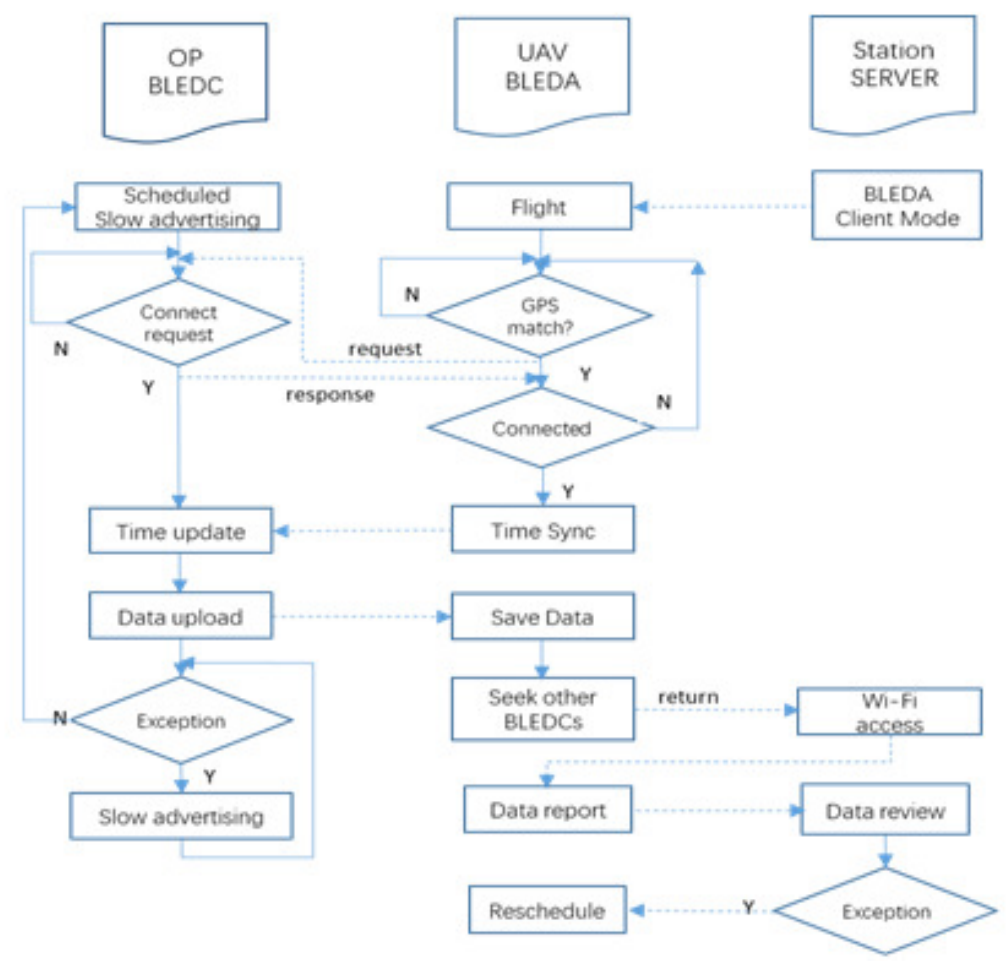

Figure 5. Routine procedure

As waypoints are applied in UAV, the path and distance is relatively fixed, and the flying speed is stable too, so it is possible that total duration of one routine procedure is countable. For instance, if UAV is scheduled to fly once an hour, duration for UAV flies from Station to the targeted forest is 5 minutes, and duration for UAV to collect all the data from BLEDCs is 10 minutes, so if BLEDCs are scheduled to start slow advertising once an hour at 5 minutes past the hour, and last 10 minutes, and once connected, not advertising in the same hour. If the schedules fulfill the requirement of the procedure, it is satisfactory and saves energy. The accurate advertising time synchronization will be developed in the future, for now, coarse schedule is enough for the system. 


\subsubsection{Exception Handle}

Station exists as a support center, it provides Wi-Fi router for UAV to access to Internet, so all the collected data from BLEDCs of every routine procedure will be uploaded to data server. As new data arrive, application in server reviews the data in a fast way first, the application checks if the degree of deviation of various sensor data using their threshold as reference, then increase the frequency of flight or start an emergence flight immediately depending on the degree of exception, in order to catch all the possible issue of surveilled forest. Similarly, BLEDC has the same mechanism to check sensor data, if emergence appears, it will keep slow advertising and waiting for connection to report data until the data return to normal and its advertising mode goes back to scheduled slow advertising. By this way, possible problem of the surveilled forest is not missed, the effectiveness of the system is kept.

\section{EXPERIMENTS}

Prototype of BLEDC is ready, which is showed in figure 6. CSR1011 is the core of BLEDC, temperature sensor is TMP112, SHT20 is alternative option as it is a combination of temperature and humidity sensors. In figure 6, a CSR1011 is connected with two TMP112s and one SHT20, and powered by a tiny CR2032 button battery. The choice of smoke dectctor is still under consideration, the current prototype is used to check feasibility of the system. BLEDA is under development, but it can be replaced by an ordinary mobile phone which is equipped with Bluetooth 4 and GPS. An Android mobile is used in experiments as it fullfills the hardware requirements and an APP is developed to implement all the procedures of BLEDA, then it is tied with camera of UAV to work as BLEDA. Three prototypes of BLEDC are hung on trees for several days, distance between each two of them is more than $100 \mathrm{~m}$ to avoid confusion of GPS information. Then their GPSs are set to DJI Phantom3 as waypoints, and each waypoint is set as point of interest, so DJI can fly to those positions and circle around and receive data. Data of different points are saved in mobile storage as seperate files, so they can ba accessed and evaluated later.

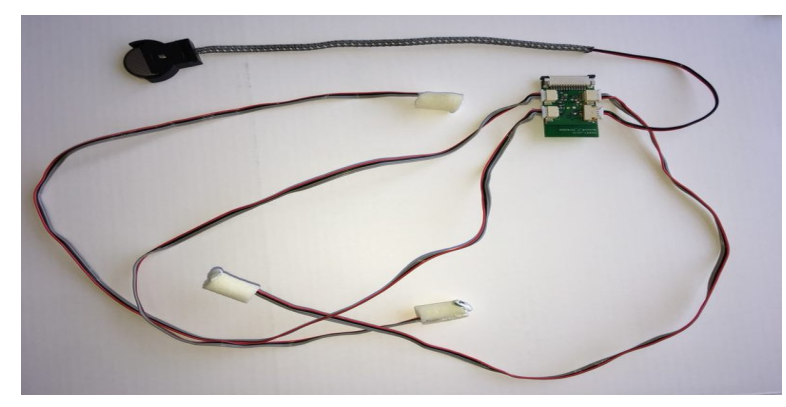

Figure 6. BLEDC prototype

Two sample data are displayed in Figure 7 and Figure 8. The data in Figure 7 is from about 20:00 of 09/21 to 18:00 of 09/22, almost a whole day of typical summer. Temperature is expressed as Celsius and humidity as percentage. In about $7 \mathrm{am}$, humidity reaches its highest and temperature in its lowest, in contrast, in midday, about $13: 00$, temperature in its highest, $55^{\circ} \mathrm{C}$ and humidity in its lowest. At other time, changes are accordant with natural law, temperature and humidity develop up and down alternatively. Figure 8 is the data of three days of rainy in a row. From afternoon of the first day, it starts raining, humidity rises and temperature drops drastically. Humidity keeps in high situation, even in the midday, the lowest percentage value of humidity is still higher than the highest ${ }^{\circ} \mathrm{C}$ value of temperature. The results are highly accordant with the real weather, which confirm that feasibility of the system. 


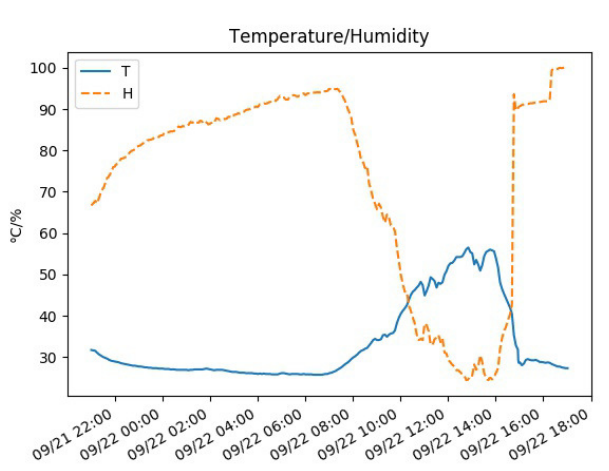

Figure 7. Normal summer day

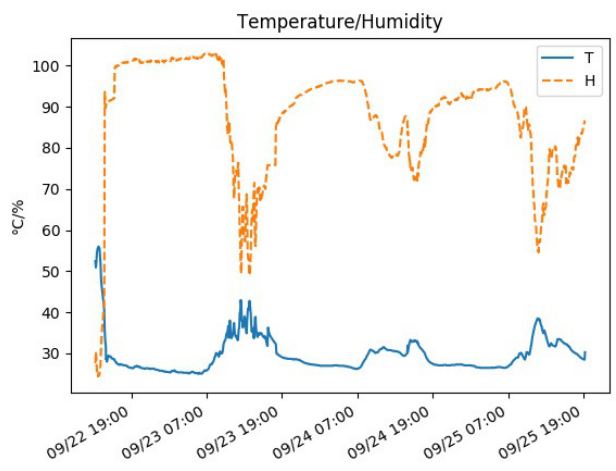

Figure 8. Three rainy days

\section{CONCLUSIONS}

UAV acts as a forest patrol worker, repeats the process again and again without fatigue to collect sensor data. Not only data from sensors, but also photos and video from action camera which is usually equipped in UAV, the combination will provide a better profile of the surveilled forest. Such a system with features of automatic, economical, one-time investment and long-term benefit, can find hidden danger of wildfire in advance, if measures can be taken before explosion of wildfire, the system is successful.

The system heavily depends on UAV which is vulnerable of severe weather, such as storm, but if the purpose of patrol is mornitoring wildfire, it is not a big issue, as wildfire usually happens in sunny hot summer not in wet days. The other weakness of UAV is its duration of flight and recharge, so the area of surveiled forest should be accordant to the ability of UAV and the schedule of flight should be planed in consideration of its battery volume.

In the future, all the hardwares should be designed resonably. First, smoke detector could be either photoelectric, which detect smoke optically, or ionization, smoke detected by physical process, no matter which one is selected, it has to be integrated to IO port of CSR1011. The current using mobile phone is much higher than the designed plan of BLEDA in cost. The third choice of BLEDA is no new hardware. As all the UAV manufactures are opening their API for developers, firmware of UAV could be adapted to implement the control and procedure of BLEDA. It will be a big save of cost. The ultimate goal of the system is prediction of wildfire which depends deeply on big data. If cooperation with local forest administaration department achieves, more and more data accumulate, a prediction pattern will emerge by data analysis and/or machine learning.

\section{REFERENCES}

[1] http://www.earth-policy.org/indicators/C56/forests_2012

[2] http://www.esri.com/services/disaster-response/wildfire

[3] A visual airborne forest fire detection patrol route planning system. (1973). Kourtz, P.H. Canadian Forestry Service, Forest Fire Research Institute, Ottawa, Ontario. Information Report FF-X-45. 34 p.

[4] An Unmanned Aircraft System for Automatic Forest Fire Monitoring and Measurement, (2012), Journal of Intelligent \& Robotic Systems, Volume 65, Number 1-4, Page 533, Luis Merino, Fernando Caballero, J. Ramiro Martínez-de-Dios, Iván Maza, Aníbal Ollero 
[5] M. Hefeeda and M. Bagheri, (2017), "Wireless Sensor Networks for Early Detection of Forest Fires," 2007 IEEE International Conference on Mobile Adhoc and Sensor Systems, Pisa, pp. 1-6. doi: 10.1109/MOBHOC.2007.4428702

[6] Antonio Molina-Pico, David Cuesta-Frau, Alvaro Araujo, Javier Alejandre, and Alba Rozas, (2016), "Forest Monitoring and Wildland Early Fire Detection by a Hierarchical Wireless Sensor Network," Journal of Sensors, vol. 2016, Article ID 8325845, 8 pages, doi: 10.1155/ 2016/ 8325845

[7] Hakan Habiboglu, Y \& Gunay, Osman \& Cetin, A. (2011). Real-time wildfire detection using correlation descriptors

[8] Yanjun Li, Zhi Wang and Yeqiong Song, (2006),"Wireless Sensor Network Design for Wildfire Monitoring, " 2006 6th World Congress on Intelligent Control and Automation, Dalian, pp. 109-113.

[9] Ahmad A. A. Alkhatib, (2014), A Review on Forest Fire Detection Techniques, International Journal of Distributed Sensor Networks Vol 10, Issue 3, 2014

[10] Brigitte Leblon, Laura Bourgeau-Chavez and Jesús San-Miguel-Ayanz (2012). Use of Remote Sensing in Wildfire Management, Sustainable Development - Authoritative and Leading Edge Content for Environmental Management, Dr. Sime Curkovic (Ed.), InTech, DOI: 10.5772/45829.

[11] Lina Tang, Guofan Shao, (2016), Drone remote sensing for forestry research and practices, Journal of Forestry Research, 2015, Volume 26, Number 4, Page 791

[12] Li, S., Lowe D., Kong X., Braun R. (2011), Wireless Sensor Network Localization Algorithm Using Dynamic Path of Mobile Beacon, APCC 2011: 17th Asia-Pacific Conference on Communications, October 2011, Sabah, Malaysia.

[13] Li S., Kong X. \& Sandrasegaran, K. (2013) "Dynamic Path of Mobile Beacon in Localization of Wireless Sensor Network" International Journal of Sensor Networks

[14] Li S., Kong X., Lowe D. (2012) "Dynamic Path of Mobile Beacon Employing Reinforcement Learning in WSN Localization". International Workshop on Data Management for Wireless and Pervasive Communications, March 2012 Japan

[15] Li S., Kong X., Lowe D. (2012) "Wireless sensor network localization with autonomous mobile beacon by path finding", International Conference on Information Science and Applications (ICISA 2012), May 2012 Suwon, S. Korea

[16] Darroudi SM, Gomez C., (2017), Bluetooth Low Energy Mesh Networks: A Survey, Sensors., 17(7): 1467.

[17] http://www.dji.com/intelligent-flight-modes/v1-doc

[18] https://www.bluetooth.com/

\section{AUTHORS}

Songsheng $\mathrm{Li}$ received his BS degree from Beijing University of Post and Communication in 1995 and MS degree from Xi'an Jiaotong University, P. R. China in 2002. He earned his Ph.D. of Engineering from University of Technology, Sydney in 2013. His research interests include Wireless Sensor Network, IOT and Data Mining.

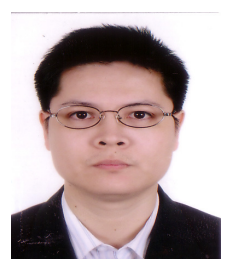

